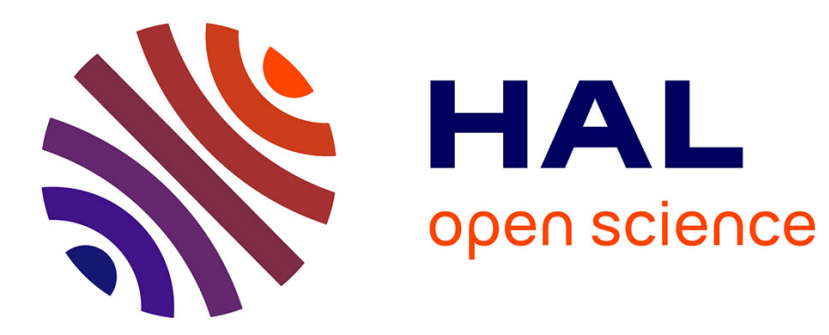

\title{
Definition of a parametric form of nonsingular Mueller matrices
}

\author{
Vincent Devlaminck, Patrick Terrier
}

\section{To cite this version:}

Vincent Devlaminck, Patrick Terrier. Definition of a parametric form of nonsingular Mueller matrices. Journal of the Optical Society of America. A Optics, Image Science, and Vision, 2008, 25 (11), pp.2636-2643. hal-00648849

\section{HAL Id: hal-00648849 \\ https://hal.science/hal-00648849}

Submitted on 8 Mar 2012

HAL is a multi-disciplinary open access archive for the deposit and dissemination of scientific research documents, whether they are published or not. The documents may come from teaching and research institutions in France or abroad, or from public or private research centers.
L'archive ouverte pluridisciplinaire $\mathbf{H A L}$, est destinée au dépôt et à la diffusion de documents scientifiques de niveau recherche, publiés ou non, émanant des établissements d'enseignement et de recherche français ou étrangers, des laboratoires publics ou privés. 


\title{
Definition of a parametric form of non singular Mueller matrices
}

\author{
V. Devlaminck \& P. Terrier \\ LAGIS - UMR CNRS 8146 \\ Université des Sciences et Technologies de Lille - 59655 France \\ e.mail : vincent.devlaminck@univ-lille1.fr
}

\section{Introduction}

Mueller matrices are very important tools for polarization optics since they describe the modifications of polarization of light after a linear interaction with a sample under interest. A $4 \times 4$ real matrix $M$ is subject to verify a set of constraints between its elements to be defined as a Mueller matrix. In the previous literature, we can distinguish between two possible definitions according to the set of constraints verified by these matrices. So, a first family of matrices transforming Stokes vectors into Stokes vectors (called "Stokes criterion") has been studied $[1,2,3,4,5,6,7]$ and there exist a complete characterization of such matrices. Givens and Kostinski [8] and van der Mee [3] conclude that if $\mathrm{G}$ stands for the Lorentz metric $\mathbf{G}=\operatorname{diag}[1,-1,-1,-1], \mathbf{M}$ corresponds to this definition of Mueller matrices if and only if the spectrum of $\mathbf{G M}^{\mathrm{T}} \mathbf{G M}$ is real and the eigenvector associated with the largest eigenvalue is a physical Stokes vector. R. Sridhar and R. Simon [1] derived also properties for set of matrices and prove for non singular matrices a canonical form $M=\mathbf{L}^{\prime} \Lambda \mathbf{L}$ where $L^{\prime}$ and $L$ are elements of the proper orthochronous Lorentz group and $\Lambda$ is a diagonal matrix.

The second family of matrices, define a Mueller matrix as an ensemble average of pure Mueller matrices or Mueller-Jones matrices ( the properties of these Mueller-Jones matrices were also extensively study and different possible interrelations for the elements of a general Mueller-Jones matrix are derived in the literature $[4,9,10,11,12]$ ). This second family of Mueller matrices was also studied [13,14,15] and Cloude [13] has proved that a $4 \times 4$ real matrix $M$ is a Mueller matrix with respect to this definition if and only if the corresponding coherency matrix constructed from $\mathrm{M}$ has only nonnegative eigenvalues.

Obviously these two families are not independent. A matrix of the second family is a matrix of the first one, but in general, the converse is not true.

How to decompose these Mueller matrices has been studied in the previous literature [1,2,16,17,18] too. $\mathrm{Lu}$ and Chipman [17] for instance, proposed a three factors decomposition widely used in the interpretation of experimental Mueller matrices $[19,20]$.

Among the tools used to analyse the mathematical properties of these matrices, most of them were derived from the linear algebra and produce algebraic representations [5] or more geometrical representation as the quaternionic formalism [21]. 
Group theory has been also used to study polarization $[13,22,23,24]$ and it is well known that non depolarizing optics can be formulated in terms of four by four representations of Lorentz group since the four components Stokes parameters may be assimilated to the Minkowskian four vectors [25].

But probably because the set of all Mueller matrices is not a group, an attempt to define a generic form for Mueller matrix is always a field of research.

Our goal in this contribution is to define a parametric form for non singular Mueller matrices. This set of matrices is not a group but a semi-group applied on a 4-dimensions space. We propose to address this problem in a space with more than 4 dimensions in order to introduce a group of transformations with the same number of degrees of freedom. After a recalling of the relations between non depolarizing matrices and Lorentz transformation group, we explain why sub-sets of $O(5,1)$, the orthogonal group associated with 6-dimensions Minkowski space, is a physical admissible solution to solve this question. Generators of this group are used to define possible expressions of an arbitrary non-singular Mueller matrix. The link with previous expressions about non depolarizing Mueller matrices is established. Eventually, the problem of decomposition of these matrices is addressed and we show that the "reverse" and "forward" decomposition concepts recently introduced [26] may be inferred from the formalism we proposed.

\section{Non depolarizing Mueller matrices}

The four-dimensional Minkowskian [27] space with $\left(\mathrm{x}^{0}, \mathrm{x}^{1}, \mathrm{x}^{2}, \mathrm{x}^{3}\right)$ coordinates - where $\mathrm{x}^{0}$ is a time-like coordinate and $\left(\mathrm{x}^{1}, \mathrm{x}^{2}, \mathrm{x}^{3}\right)$ are space-like coordinates - is the four vectors space associated with the Minkowski metric. This metric is defined by the metric tensor $g_{\mu v}$ with the signature $(1,-1,-1,-1)$ and the definition of the "length" of a 4-vector $\mathrm{x}$ can be written as

$$
[x, x]=g_{\mu \nu} x^{\mu} x^{\nu}
$$

where $[.,$.$] stands for the indefinite scalar product [3,28,29]$ and where the classical implicit summations (Einstein notation ) extend over all 4 components.

First, we recall here the connections between linear transformations of the Minkowski space which preserve the "length" of the 4-vectors and the non singular-nondepolarizing Mueller matrices. Those matrices describe elements that convert completely polarized light into completely polarized light and they have equivalent nonsingular Jones matrices [17]. It is well known [2,3,4] that a real invertible $4 \times 4$ matrix is a non-depolarizing Mueller matrix if and only if it is a positive multiple of an element within the group of restricted linear transformations of the Minkowski space which preserve the length of the Stokes 4-vectors $S=\left(S_{0} S_{1} S_{2} S_{3}\right)$.

The set of transformations which preserve the length of the 4-vectors is known as the Lorentz group. Combining length conservation equation and the basic relation between a Lorentz transformation $\Gamma$ and the input and output 4-vectors $\mathrm{x}_{\text {out }}^{\mu}=\Gamma_{\lambda}^{\mu} \mathrm{x}_{\lambda}^{\text {in }}$, the condition on Lorentz transformation can be reformulated as :

$$
\mathrm{g}_{\mu \nu} \Gamma_{\lambda}^{\mu} \Gamma_{\sigma}^{\nu}=\mathrm{g}_{\lambda \sigma} \quad \text { or } \quad \Gamma_{\lambda}^{\mu} \Gamma_{\sigma}^{\nu} \mathrm{g}^{\lambda \sigma}=\mathrm{g}^{\mu \nu}
$$

We note that, setting $\lambda=\sigma$ in the first expression, we find the condition : 
$\left(\Gamma_{0}^{0}\right)^{2}-\sum_{\mathrm{i}}\left(\Gamma_{0}^{\mathrm{i}}\right)^{2}=1 \quad$ which implies $\left(\Gamma_{0}^{0}\right)^{2} \geqslant 1$ and taking the determinant on both sides of Eq. (2), we obtain $\operatorname{det}(\Gamma)^{2}=1$. It follows that the Lorentz group is the union of four disconnected pieces [30]. The restriction to those transformations which are continuously connected to the identity transformation (called the proper orthochronous Lorentz transformations and denoted $S O(3,1)_{e}$ as the identity component of $S O(3,1)$, the associated special orthogonal group. ) requires that $\left(\Gamma_{0}^{0}\right) \geqslant 1$ and $\operatorname{det}(\Gamma)=1$.

Under this restriction, the nonsingular nondepolarizing Mueller matrices are the positive multiples of Lorentz group matrices with positive left upper corner element and positive determinant. These group of matrices is the nonsingular nondepolarizing matrices group with respect to the definition of Mueller matrices as an ensemble average of pure Mueller matrices. The nonsingular nondepolarizing matrices with respect to the "Stokes criterion" definition, are the positive multiples of Lorentz group matrices with positive left upper corner element. Then, corresponding sub-group is given $[30]$ by $\left\{S O(3,1)_{e}\right\}$ $\cup\left\{\mathrm{G} . S O(3,1)_{e}\right\}$ with $\mathrm{G}=\operatorname{diag}(1,-1,-1,-1)$. So, the characterisation of matrices of $\mathrm{S} O(3,1)_{e}$ completely describes the two sub-groups.

Two examples of transformations of $S O(3,1)_{e}$ are the 3-dimensions rotations and the Lorentz boost. Rotations in the 3 spatial dimensions are of the form:

$$
R=\left(\begin{array}{cc}
1 & {[0]} \\
{[0]^{\mathrm{T}}} & {[\mathrm{r}]}
\end{array}\right)
$$

where $\mathrm{r}$ denotes ordinary $3 \times 3$ rotation matrices and [0] is for $\left[\begin{array}{lll}0 & 0 & 0\end{array}\right]$ vector. As the 3dimensions rotations depend on 3 parameters (Euler angles $\phi, \theta, \psi$ for instance), $\mathrm{R}=\mathrm{R}(\phi, \theta, \psi)$ denotes the corresponding rotation in the Lorentz group with: $\mathrm{R}(\phi, \theta, \psi)=\mathrm{R}_{3}(\phi) \mathrm{R}_{2}(\theta) \mathrm{R}_{3}(\psi)$. Thus in terms of the Euler angles, every rotation can be decomposed into a product of elementary rotations around the fixed axes of $x^{2}$ and $x^{3}$.

The second example of these Lorentz transformations is the Lorentz boost. Such transformations mix space-like coordinates with the time-like coordinate. A simple example of these boosts is the Lorentz boost along the $\mathrm{x}^{3}$ coordinate with the associated matrix $L_{3}(u)$ (corresponding expressions for the boost along the $x^{1}$ and $x^{2}$ coordinate can be derived from Ref. [25] ):

$$
\mathrm{L}_{3}(\mathrm{u})=\left(\begin{array}{cccc}
\cosh (\mathrm{u}) & 0 & 0 & \sinh (\mathrm{u}) \\
0 & 1 & 0 & 0 \\
0 & 0 & 1 & 0 \\
\sinh (\mathrm{u}) & 0 & 0 & \cosh (\mathrm{u})
\end{array}\right)
$$

It is straightforward to prove $[3,22]$ that these Lorentz transformations matrices may be regarded as the translation into the four by four formalism of Jones matrices: Jones matrices of elliptical retarders (or phase shifters) and elliptical diattenuators (or partial polarizers) respectively.

Since a general element $\Gamma$ of the proper orthochronous Lorentz group can be uniquely written in the factorized form ( Ref. [27] contains a generic form of this factorization. Eq. (5) can be derived from the prof of this property by just noting that the rotation which brings an arbitrary vector to the $\mathrm{z}$ axis is the result of a first rotation around the $\mathrm{z}$ axis by angle $\alpha$ followed by a rotation around the y axis by angle $\beta$, see [27] pp.11-12 for notation): 


$$
\Gamma=\mathrm{R}(\phi, \theta, \psi) \mathrm{L}_{3}(\mathrm{u}) \mathrm{R}^{-1}(\alpha, \beta, 0)
$$

a non-depolarizing Mueller matrix $\mathrm{M}$ (with respect to second definition family), proportional (with a positive constant k) to a Lorentz transformation $\Gamma$, may be factorized in two terms, a pure rotation and a pure Lorentz boost and from Eq.(5) we have:

$$
\mathrm{M}=\mathrm{k}\left[\mathrm{R}(\phi, \theta, \Psi) \mathrm{L}_{3}(0) \mathrm{R}^{-1}(\alpha, \beta, 0)\right]\left[\mathrm{R}(\alpha, \beta, 0) \mathrm{L}_{3}(\mathrm{u}) \mathrm{R}^{-1}(\alpha, \beta, 0)\right]
$$

where the pure rotation and pure Lorentz boost are the first and the second bracketed factor, respectively. And arbitrary elements of the group can be written as the product of those two matrices but for suitable $\mathrm{u}, \alpha, \beta, \varphi, \theta$ and $\psi$ since the matrix multiplication is not commutative.

These non-depolarizing matrices have only 7 degrees of freedom [31]. So, there are nine relations between the 16 matrix elements. These nine relations have already been derived explicitly in [32,33] for instance. This result is obvious when we observe Eq. (6) but it could be also predicted as can be seen as follows: a general $4 \times 4$ real matrix has 16 arbitrary elements but Eq.(2) contains 10 independent constraints. The result is then only 6 degrees of freedom for a Lorentz transformation. For non-depolarizing Mueller matrices, one degree of freedom is added by the proportionality constant $\mathrm{k}$ of Eq.(6).

\section{Non singular Mueller matrices}

This approach is very interesting to define a mathematical framework to investigate the most general expression of non singular Mueller matrices. This set of matrices is not a group ( in fact it is a semi-group) - the inverse matrix of a pure depolarizer for instance is not a Mueller matrix - but this difficulty may be overcome if we consider a group of transformations continuously connected to the identity transformation and preserving a $\mathrm{N}$-vectors norm such as the proper Lorentz transformations with the 4-vectors of the Minkowski space.

The problems with such approach are : how do we determine $\mathrm{N}$, the space dimension and how do we chose the appropriate norm? For a $\mathrm{N}$ dimensions space, the general rule gives the number of degrees of freedom $\mathrm{D}=\mathrm{N}^{2}-\mathrm{N}(\mathrm{N}+1) / 2=\mathrm{N}(\mathrm{N}-1) / 2$. So it is easy to find out that with $\mathrm{N}=6$ we reach the total of 15 degrees of freedom ( 36 elements but 21 independent constraints) which is the number of degrees of freedom of a general Mueller matrix (after normalization by the first coefficient for instance). A similar approach was already used by Cloude [13] to develop an homomorphism between $\mathrm{SU}(4)$ (the Special Unitary group) and $\mathrm{O}^{+}(6)$ (the Real Orthogonal group) used for describing the interaction of polarized waves with targets.

Following a similar approach proposed in [13,22], we introduce a 6-dimensions space in order to deal with this problem. This 6-dimensions space has 6 coordinates $\left(\mathrm{x}^{0}, \mathrm{x}^{1}, \mathrm{x}^{2}, \mathrm{x}^{3}, \mathrm{x}^{4}, \mathrm{x}^{5}\right)$. The proposed space should contain a Minkowskian subspace associated with the four first components for instance. Then, this subspace can be assimilated with the polarization space (it means $\left.\left(x^{0}, x^{1}, x^{2}, x^{3}\right)=\left(S_{0} S_{1} S_{2} S_{3}\right)\right)$. An associated metric is defined by the metric tensor $g_{\mu v}$ with the signature $\left(1,-1,-1,-1, \mathrm{~g}_{44}\right.$, $\mathrm{g}_{55}$ ) and the study of the previous paragraph allows to maintain that the linear 
transformations of this space which preserve the length of these 6-vectors have 15 degrees of freedom.

In order to find out the sign of the both unknown values of the metric tensor, we note that setting $\mu=v=0$ in the second equation (2), we obtain the condition:

$$
\left(\Gamma_{0}^{0}\right)^{2}-\sum_{\mathrm{i}=1}^{3}\left(\Gamma_{\mathrm{i}}^{0}\right)^{2}+\mathrm{g}^{44}\left(\Gamma_{4}^{0}\right)^{2}+\mathrm{g}^{55}\left(\Gamma_{5}^{0}\right)^{2}=1
$$

We consider now the case of unpolarized incident state. The exiting state is determined only by the first column of the Mueller matrix and the degree of polarisation of this light resulting from the unpolarized input is called [17,34] the polarizance P. This polarizance is given by:

$$
\mathrm{P}=\frac{\sqrt{\left(\Gamma_{1}^{0}\right)^{2}+\left(\Gamma_{2}^{0}\right)^{2}+\left(\Gamma_{3}^{0}\right)^{2}}}{\Gamma_{0}^{0}}
$$

Combining Eqs. (7)- (8), we obtain the expression:

$$
\mathrm{P}^{2}=1-\frac{1-\mathrm{g}^{44}\left(\Gamma_{4}^{0}\right)^{2}-\mathrm{g}^{55}\left(\Gamma_{5}^{0}\right)^{2}}{\left(\Gamma_{0}^{0}\right)^{2}}
$$

As the degree of polarization of the exiting beam cannot be greater than 1 , it is clear from (9) that we must have $\mathrm{g}^{44}=\mathrm{g}^{55}=-1$ in order to deal with a physical meaning solution. So, we are now considering the group $O(5,1)$ and more precisely its identity component $S O(5,1)$ e. Matrices with unit determinant and positive left upper corner element are the $S O(5,1)_{\text {e }}$ subgroup elements. Matrices with unit square determinant and positive left upper corner element are the $\left\{S O(5,1)_{e}\right\} \cup\left\{\mathrm{G} . S O(5,1)_{e}\right\}$ subgroup elements. As with $S O(3,1)$ e and the nondepolarizing matrices, the characterisation of matrices of $S O(5,1)_{e}$ completely describes the two sub-groups. But what was impossible to deal in the 4-dimensions space with the $S O(3,1)_{\mathrm{e}}$ transformations (change the value of the norm of the Stokes vector) will be now possible in the full space where this subspace is included and the $4 \times 4$ matrix (associated with the polarization space $\left(\mathrm{x}^{0}, \mathrm{x}^{1}, \mathrm{x}^{2}, \mathrm{x}^{3}\right)$ will be always a physical Mueller matrix.

As $S O(5,1)_{e}$ is a matrix Lie group with 15 degrees of freedom, we can focus our attention on the 15 generators of the group rather than the infinite number of group elements. These generators are elements of the Lie algebra [35] of the group $S O(5,1)_{e}-$ The Lie algebra of a matrix Lie group $L G$ is the set of all matrices $\mathrm{X}$ such that $\mathrm{e}^{\mathrm{itX}}$ is in $L G$ for all real number $\mathrm{t}$ - Once the generators are known, the group elements can all be determined since this set of generators is a basis for the Lie algebra that is also a vector space. Six of these generators may be regarded as the translation of well known Lorentz group generators in the 6-dimensions space. We can dissociate these 6 generators in terms of more familiar quantities: 3 rotations generators: $J_{23}, J_{13}, J_{12}$ stand for the generators of rotation of the $\left(\mathrm{x}^{1}, \mathrm{x}^{2}, \mathrm{x}^{3}\right)$ space-like components; 3 boost generators: $\mathrm{J}_{01}, \mathrm{~J}_{02}, \mathrm{~J}_{03}$ stand for the generators of boosts mixing space-like coordinate $\mathrm{x}^{\mathrm{j}}$ $(\mathrm{j}=1,2,3)$ with the time-like coordinate $\mathrm{x}^{0}$. In the 6 dimensions space, we have now two other boost generators $J_{04}, J_{05}$ and 7 other rotation generators $J_{14}, J_{24}, J_{34}, J_{15}, J_{25}, J_{35}$, $\mathrm{J}_{45}$.

Eq.(10) shows examples of these generators and the associated matrices. The generators 
$\left(\mathrm{J}_{01}, \mathrm{~J}_{02}, \mathrm{~J}_{04}, \mathrm{~J}_{05}\right)$ may be deduced from the expression of $\mathrm{J}_{03},\left(\mathrm{~J}_{34}, \mathrm{~J}_{45}, \mathrm{~J}_{14}, \mathrm{~J}_{25}\right)$ from $\mathrm{J}_{12}$ and $\left(\mathrm{J}_{24}, \mathrm{~J}_{35}, \mathrm{~J}_{15}\right)$ from $\mathrm{J}_{13}$.

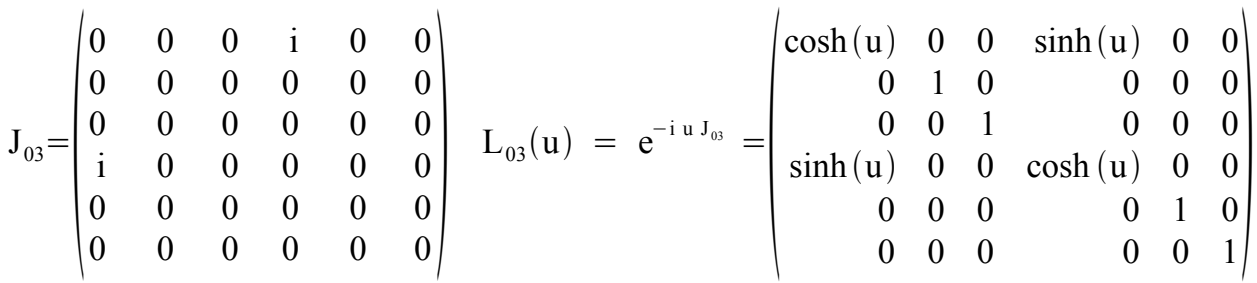

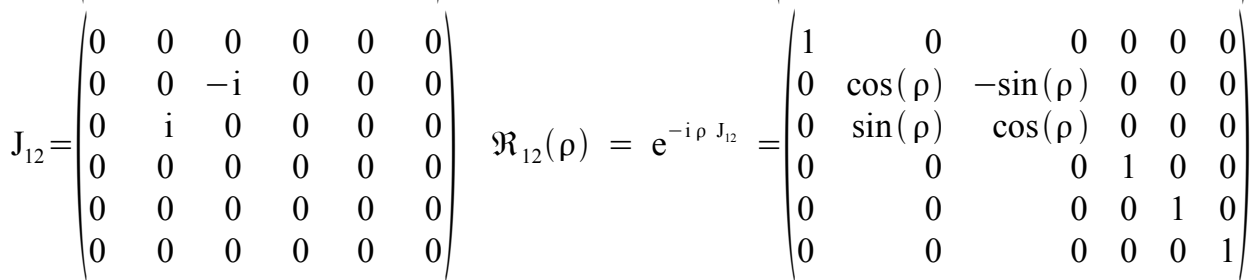

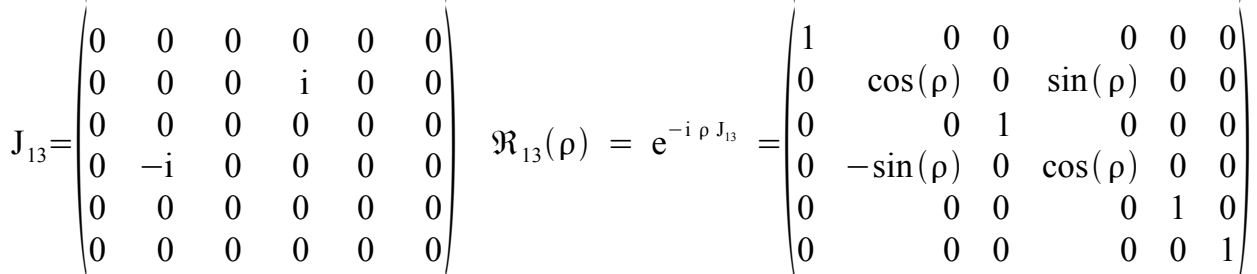

$$
\begin{aligned}
& \mathrm{J}_{23}=\left(\begin{array}{rrrrrr}
0 & 0 & 0 & 0 & 0 & 0 \\
0 & 0 & 0 & 0 & 0 & 0 \\
0 & 0 & 0 & -\mathrm{i} & 0 & 0 \\
0 & 0 & \mathrm{i} & 0 & 0 & 0 \\
0 & 0 & 0 & 0 & 0 & 0 \\
0 & 0 & 0 & 0 & 0 & 0
\end{array}\right) \quad R_{23}(\rho)=\mathrm{e}^{-\mathrm{i} \rho \mathrm{J}_{23}}=\left(\begin{array}{rrrrrr}
1 & 0 & 0 & 0 & 0 & 0 \\
0 & 1 & 0 & 0 & 0 & 0 \\
0 & 0 & \cos (\rho) & -\sin (\rho) & 0 & 0 \\
0 & 0 & \sin (\rho) & \cos (\rho) & 0 & 0 \\
0 & 0 & 0 & 0 & 1 & 0 \\
0 & 0 & 0 & 0 & 0 & 1
\end{array}\right)
\end{aligned}
$$

According to the matrix Lie group theory, we can deduce a formal expression for an arbitrary element $\mathrm{T}$ of $S O(5,1)_{e}$ (with the Einstein notation):

$$
\mathrm{T}=\mathrm{e}^{-\frac{\mathrm{i}}{2} \alpha_{\mathrm{mn}} \mathrm{J}_{\mathrm{mn}}}
$$

where $\alpha_{m n}$ is a 15 parameters anti-symmetric second rank tensor (15 parameters corresponding to the 15 degrees of freedom).

So, an arbitrary non-singular Mueller matrix is given by the $4 \times 4$ sub-matrix extracted from the T matrix of Eq.(11). This first expression for a non-singular Mueller matrix is not necessarily the most practical one for analysing the physical phenomena expressed by these matrices. For instance, the previous expression of non-depolarizing matrices derived from the Lorentz transformations can not be readily extracted from this expression since $e^{(A+B)} \neq e^{A} e^{B}$ for non commuting matrices $(A B-B A=[A, B] \neq 0$, where $[\mathrm{A}, \mathrm{B}]$ operator is termed the commutator or brackets of $\mathrm{A}, \mathrm{B}$ matrices ). More useful expression can be derived from the commutation relations of the generators of this Lie algebra.

Commutation rules associated with this Lie algebra are given by two sets of relations (explicit calculations of the matrix products give the following results) : 


$$
\begin{aligned}
& \begin{array}{lll}
{\left[\mathrm{J}_{12}, \mathrm{~J}_{13}\right]=-\mathrm{iJ}_{23}} & {\left[\mathrm{~J}_{12}, \mathrm{~J}_{23}\right]=\mathrm{i} \mathrm{J}_{13}} & {\left[\mathrm{~J}_{13}, \mathrm{~J}_{23}\right]=-\mathrm{i} \mathrm{J}_{12}} \\
{\left[\mathrm{~J}_{04}, \mathrm{~J}_{05}\right]=-\mathrm{iJ}_{45}} & {\left[\mathrm{~J}_{05}, \mathrm{~J}_{45}\right]=\mathrm{iJ}_{04}} & {\left[\mathrm{~J}_{04}, \mathrm{~J}_{45}\right]=-\mathrm{iJ}_{05}}
\end{array} \\
& {\left[\mathrm{~J}_{\mathrm{h} 4}, \mathrm{~J}_{\mathrm{km}}\right]=-\mathrm{i} \epsilon_{\mathrm{hkm}} \mathrm{J}_{\mathrm{m} 4} \quad \mathrm{k}, \mathrm{h}, \mathrm{m} \in\{1,2,3\}} \\
& {\left[\mathrm{J}_{\mathrm{h} 5}, \mathrm{~J}_{\mathrm{km}}\right]=-\mathrm{i} \epsilon_{\mathrm{hkm}} \mathrm{J}_{\mathrm{m} 5} \quad \mathrm{k}, \mathrm{h}, \mathrm{m} \in\{1,2,3\}} \\
& {\left[\mathrm{J}_{0 \mathrm{~h}}, \mathrm{~J}_{\mathrm{km}}\right]=-\mathrm{i} \epsilon_{\mathrm{hkm}} \mathrm{J}_{0 \mathrm{~m}} \quad \mathrm{k}, \mathrm{h}, \mathrm{m} \in\{1,2,3\}}
\end{aligned}
$$

where $\epsilon_{\mathrm{hkm}}$ is the totally anti-symmetric unit tensor of rank 3 .

The two sets of relations (12) \& (13) show that these generators are closed under brackets and immediate consequences of the Lie algebra associated with the generators of Eq. (13) are for $\mathrm{j} \in\{1,2,3\}$ :

$$
\begin{aligned}
& \mathfrak{R J}_{\mathrm{j} 4} \Re^{-1}=\mathrm{J}_{\mathrm{k} 4} \Re_{\mathrm{j}}^{\mathrm{k}} \\
& \mathfrak{R J}_{\mathrm{j} 5} \Re^{-1}=\mathrm{J}_{\mathrm{k} 5} \Re_{\mathrm{j}}^{\mathrm{k}} \\
& \mathfrak{R J}_{0 \mathrm{j}} \Re^{-1}=\mathrm{J}_{0 \mathrm{k}} \Re_{\mathrm{j}}^{\mathrm{k}}
\end{aligned}
$$

where $\mathfrak{R}$ is the 6-dimensions matrix ( $\mathfrak{R}_{\mathrm{j}}^{\mathrm{k}}$ is the $j$-th element of the $k$-th row of $\Re$ ) of any 3-rotation of $\left(\mathrm{x}^{1}, \mathrm{x}^{2}, \mathrm{x}^{3}\right)$ space-like coordinates and we have $\mathfrak{R}(\alpha, \beta, \gamma)=\mathfrak{R}_{12}(\alpha) \mathfrak{R}_{13}(\beta) \mathfrak{R}_{12}(\gamma)$ where $\mathfrak{R}_{\mathrm{ij}}$ are defined in Eqs. (10) . It follows then from Eqs.(14) and the first set of Eqs. (12) that

$$
\begin{aligned}
& \mathrm{J}_{\overrightarrow{\mathrm{n}} 4}=\Re \mathrm{J}_{34} \Re^{-1}=\mathrm{J}_{\mathrm{k} 4} \mathfrak{R}(\alpha, \beta, 0)_{3}^{\mathrm{k}}=\mathrm{J}_{\mathrm{k} 4} \mathrm{n}^{\mathrm{k}} \\
& \mathrm{J}_{\overrightarrow{\mathrm{n}} 5}=\mathfrak{R J}_{35} \Re^{-1}=\mathrm{J}_{\mathrm{k} 5} \mathfrak{R}(\alpha, \beta, 0)_{3}^{\mathrm{k}}=\mathrm{J}_{\mathrm{k} 5} \mathrm{n}^{\mathrm{k}} \\
& \mathrm{J}_{0 \overrightarrow{\mathrm{n}}}=\Re \mathrm{J}_{03} \Re^{-1}=\mathrm{J}_{0 \mathrm{k}} \mathfrak{R}(\alpha, \beta, 0)_{3}^{\mathrm{k}}=\mathrm{J}_{0 \mathrm{k}} \mathrm{n}^{\mathrm{k}}
\end{aligned}
$$

where $n^{k}$ are the components of any $\left(x^{1}, x^{2}, x^{3}\right)$ arbitrary spatial directions $\vec{n}$ defined by the rotation $\mathfrak{R}(\alpha, \beta, 0)$ which brings basis vector of axis $x^{3}$ to $\vec{n}(\alpha, \beta)$.

A similar expression for the rotation of the spatial components $\left(\mathrm{x}^{1}, \mathrm{x}^{2}, \mathrm{x}^{3}\right)$ can be derived from the first set of Eqs. (12):

$$
\mathrm{J}_{\overrightarrow{\mathrm{n}}}=\Re \mathrm{J}_{12} \mathfrak{R}^{-1}=\mathrm{J}_{\mathrm{k}} \mathfrak{R}(\alpha, \beta, 0)_{3}^{\mathrm{k}}=\mathrm{J}_{\mathrm{k}} \mathrm{n}^{\mathrm{k}}
$$

with $\mathrm{J}_{1}=\mathrm{J}_{23}, \mathrm{~J}_{2}=\mathrm{J}_{12}$ and $\mathrm{J}_{3}=\mathrm{J}_{13}$.

Eq. (15) shows that $\left\{\mathrm{J}_{\mathrm{j} 4}\right\},\left\{\mathrm{J}_{\mathrm{j} 5}\right\}$ and $\left\{\mathrm{J}_{0 \mathrm{j}}\right\}$ form basis for the generators $\mathrm{J}_{\overrightarrow{\mathrm{n}} 4}, \mathrm{~J}_{\overrightarrow{\mathrm{n}} 5}$ and $J_{0 \vec{n}}$ respectively and Eq. (16) shows that $J_{23}, J_{12}$ and $J_{13}$ form a basis for the generators $\mathrm{J}_{\overrightarrow{\mathrm{n}}}$. Thus, from the previous properties and the elementary matrix identity: $\mathfrak{R} \mathrm{e}^{-\mathrm{i} \alpha \mathrm{G}} \mathfrak{R}^{-1}=\mathrm{e}^{-\mathrm{i} \alpha\left(\mathfrak{R} \mathrm{G} \Re^{-1}\right)}$, T may be decomposed as a product of five $6 \times 6$ matrices (in whatever order) for suitable parameters

$$
\begin{aligned}
& \mathrm{T}_{\mathrm{R}}=\left[\mathfrak{R}(\phi, \theta, 0) \Re_{12}(\psi) \mathfrak{R}^{-1}(\phi, \theta, 0)\right] \quad \mathrm{T}_{\mathrm{D}}=\left[\mathfrak{R}(\alpha, \beta, 0) \mathrm{L}_{03}(\mathrm{u}) \mathfrak{R}^{-1}(\alpha, \beta, 0)\right] \\
& \mathrm{T}_{\Delta 1234}=\left[\mathfrak{R}(\gamma, \delta, 0) \mathfrak{R}_{34}(\mu) \mathfrak{R}^{-1}(\gamma, \delta, 0)\right] \mathrm{T}_{\Delta 1235}=\left[\mathfrak{R}(\epsilon, \eta, 0) \mathfrak{R}_{35}(\nu) \mathfrak{R}^{-1}(\epsilon, \eta, 0)\right] \\
& \mathrm{T}_{\Delta 045}=\left[\mathrm{L}_{04}(\mathrm{v}) \mathfrak{R}_{45}(\mathrm{\tau}) \mathrm{L}_{05}(\mathrm{w})\right]
\end{aligned}
$$


with $T_{R}$ resulting from Eq.(16), $T_{D}$ from the third equation of Eqs. (15), $T_{\Delta 1234}$ from the first equation of Eqs. (15), $\mathrm{T}_{\Delta 1235}$ from the second equation of Eqs. (15) and $\mathrm{T}_{\Delta 045}$ from the second line of Eqs. (12). So, a general transformation $\mathrm{T}$ of $S O(5,1)_{e}$ may be regarded as a combination of space-like coordinates rotation in a plane specified by a unit vector

$\vec{n}(\phi, \theta)$ and the $x^{3}$ axis and with an angle of rotation $\psi$, a boost with $u$ as parameter mixing the time-like coordinates with a $\left(x^{1}, x^{2}, x^{3}\right)$ spatial direction $\vec{n}(\alpha, \beta)$, a rotation in a plane specified by a unit vector $\vec{n}(\gamma, \delta)$ and $x^{4}$ axis and an other one in a plane specified by a unit vector $\vec{n}(\epsilon, \eta)$ and $x^{5}$ axis. These latter rotations are with angles of rotation $\mu$ and $v$ respectively. The last term is formed by the closed set of generators $\mathrm{J}_{04}, \mathrm{~J}_{05}$ and $\mathrm{J}_{45}$.

\section{Non singular Mueller matrix decomposition}

It is then a straightforward calculation from Eq.(17) to show that the corresponding submatrices associated with the polarization space (or Mueller matrices) may be regarded as a product of three matrices $M_{R}, M_{D}$ and $M_{\Delta} . M_{R}$ (retardance ) and $M_{D}$ (diattenuation), related to $T_{R}$ and $T_{D}$ are the non depolarizing Mueller matrices of the decomposition and $\mathrm{M}_{\Delta}$ ( related to $\mathrm{T}_{\Delta 1234}, \mathrm{~T}_{\Delta 1235}$ and $\mathrm{T}_{\Delta 045}$ and denoted $\mathrm{T}_{\Delta}$ ) stands for the depolarizing matrix.

The first part $\left(T_{R} T_{D}\right.$ matrices) of the decomposition may be regarded as the translation of Eq.(6) in the 6-dimensions space. It is straightforward from definitions of $\mathrm{L}_{30}$ and rotation matrices to prove that the product of these matrices has the form:

$$
\mathrm{T}_{\mathrm{R}} \mathrm{T}_{\mathrm{D}}=\left(\begin{array}{cc}
\mathrm{M}_{\mathrm{R}} \mathrm{M}_{\mathrm{D}} & 0 \\
0 & \mathrm{I}_{2}
\end{array}\right) \quad \mathrm{T}_{\mathrm{D}^{\prime}} \mathrm{T}_{\mathrm{R}^{\prime}}=\left(\begin{array}{cc}
\mathrm{M}_{\mathrm{D}^{\prime}} \mathrm{M}_{\mathrm{R}^{\prime}} & 0 \\
0 & \mathrm{I}_{2}
\end{array}\right)
$$

where $M_{R}$ and $M_{D}$ are matrices defined by Eqs.(6) and $I_{2}$ is for the identity matrix of order two.

The second part of the decomposition formed by the product of $\mathrm{T}_{\Delta 1234}, \mathrm{~T}_{\Delta 1235}$ and $\mathrm{T}_{\Delta 045}$ matrices taken in whatever order, is written as

$$
\mathrm{T}_{\Delta}=\left(\begin{array}{cc}
\mathrm{M}_{\Delta} & \mathrm{X} \\
\mathrm{Y} & \mathrm{Z}
\end{array}\right)
$$

With the expressions of matrices of Eq. (18)-(19), a sub-matrix associated with the polarization space is then the product of $M_{R}, M_{D}$ and $M_{\Delta}$ in whatever order. The third factor $\mathrm{M}_{\Delta}$ standing for the depolarizing matrix has not a so straightforward interpretation as the first ones because of the commutation relations of the corresponding generators.

\section{5 - Decomposition of depolarizing Mueller matrices}

The great difference between the set of generators of the non depolarizing part $\left(\mathrm{J}_{01}, \mathrm{~J}_{02}\right.$, $\mathrm{J}_{03}, \mathrm{~J}_{12} \quad \mathrm{~J}_{13}$ and $\left.\mathrm{J}_{23}\right)$ and the set of generators of the depolarizing part $\left(\mathrm{J}_{04}, \mathrm{~J}_{05}, \mathrm{~J}_{45}, \mathrm{~J}_{14}, \mathrm{~J}_{24}\right.$, $\mathrm{J}_{34}, \mathrm{~J}_{15} \mathrm{~J}_{25}$ and $\mathrm{J}_{35}$ ) comes from closure properties under the bracket operation. The 
second set is not closed ( $\left[\mathrm{J}_{14}, \mathrm{~J}_{34}\right]=-\mathrm{i} . \mathrm{J}_{13}$, for instance) and mixes indices from the polarization space (that is $\left.\left(\mathrm{x}^{0}, \mathrm{x}^{1}, \mathrm{x}^{2}, \mathrm{x}^{3}\right)\right)$ with the two other indices. In fact, we also have a closure relation with respect to indices 04, 05 and 45 ( second set of relations from Eqs.(12)) and the problem is mainly concentrated on the 6 others generators. Immediate consequences are: we may have a non depolarizing part in the $\mathrm{M}_{\Delta}$ matrices and the Mueller matrix related to the product of $T_{\Delta 1}$ and $T_{\Delta 2}$ is not necessary the product of the two matrices $\mathrm{M}_{\Delta 1}$ and $\mathrm{M}_{\Delta 2}$ extracted from $\mathrm{T}_{\Delta 1}$ and $\mathrm{T}_{\Delta 2}$ respectively (but it is a Mueller matrix).

If we used for instance the previous decomposition of $\mathrm{T}_{\Delta}$ as a product of $\mathrm{T}_{\Delta 1234}, \mathrm{~T}_{\Delta 1235}$ and $\mathrm{T}_{\Delta 045}$, the associated Mueller matrices have the following expressions (from Eq. (10) and (17)):

$$
\mathrm{M}_{\Delta 1234}=\left(\begin{array}{cc}
1 & {[0]} \\
{[0]^{\mathrm{T}}} & \mathrm{m}_{\Delta 1234}
\end{array}\right) \quad \mathrm{M}_{\Delta 1235}=\left(\begin{array}{cc}
1 & {[0]} \\
{[0]^{\mathrm{T}}} & \mathrm{m}_{\Delta 1235}
\end{array}\right) \quad \mathrm{M}_{\Delta 045}=\left(\begin{array}{cc}
\mathrm{a} & {[0]} \\
{[0]^{\mathrm{T}}} & \mathrm{I}_{3}
\end{array}\right)
$$

where $\mathrm{m}_{\Delta 1234}$ and $\mathrm{m}_{\Delta 1235}$ are symmetric matrices according to the corresponding expressions of $\mathfrak{R}_{34}$ and $\mathfrak{R}_{35}$ matrices. $\mathbf{M}_{\Delta 1234}$ and $\mathbf{M}_{\Delta 1235}$ have the same kind of expression but with different parameters. Their product is not a symmetric matrix in the general case. So if we decompose this matrix by a polar decomposition (proposed by Lu \& Chipman [17] ) in order to symmetrize it, a rotation matrix $M_{R}$ will be extracted.

Nevertheless, instead of considering the matrices $\mathrm{T}_{\Delta 1234}, \mathrm{~T}_{\Delta 1235}$ and $\mathrm{T}_{\Delta 045}$ and their Mueller matrix counterparts, it is possible to directly construct a symmetric expression considering the matrices $T_{\Delta 12345}$ generated by the following set of generators $\left\{J_{14}, J_{24}\right.$, $\left.\mathrm{J}_{34}, \mathrm{~J}_{15} \mathrm{~J}_{25}, \mathrm{~J}_{35}\right\}$. The associated Mueller matrices have the following expression (see appendix A for the proof):

$$
\mathrm{M}_{\Delta 12345}=\left(\begin{array}{cc}
1 & {[0]} \\
{[0]^{\mathrm{T}}} & \mathrm{m}_{\Delta 12345}
\end{array}\right)
$$

where $\mathrm{m}_{\Delta 12345}$ is a symmetric matrix. From the commutation relations of these generators - Eqs. (13) - and a similar approach previously used to derived Eqs. (17), it is possible to prove that $\mathrm{T}_{\Delta 12345}$ has the following expression:

$$
\begin{aligned}
& \mathrm{T}_{\Delta 12345}=\left[\mathfrak{R}_{12}(\epsilon) \mathfrak{R}_{13}(\eta)\right]\left[\mathfrak{R}_{23}(\phi)\right.\left.\mathfrak{R}_{45}(\psi)\right] \mathfrak{R}_{34}(\mu) \mathfrak{R}_{25}(\nu) \ldots \\
& \ldots \ldots\left[\mathfrak{R}_{23}(\phi) \mathfrak{R}_{45}(\psi)\right]^{-1}\left[\mathfrak{R}_{12}(\epsilon) \mathfrak{R}_{13}(\eta)\right]^{-1}
\end{aligned}
$$

Since the expressions of Mueller matrices associated with $\mathfrak{R}_{34}$ and $\mathfrak{R}_{25}$ are already in a diagonalized form (straightforward from the definition of $\mathfrak{R}_{34}(\mu)=\mathrm{e}^{-\mathrm{i} \mu \mathrm{J}_{34}}$ and $\left.\mathfrak{R}_{25}(\nu)=\mathrm{e}^{-\mathrm{i} v \mathrm{~J}_{25}}\right)$, with eigenvalues for the corresponding $3 \times 3$ m-matrices $\{1,1$, $\cos (\mu)\}$ and $\{1, \cos (v), 1\}$ respectively, it results from Eq. (22) that the eigenvalues of $\mathrm{m}_{\Delta 12345}$ are given by $\{1, \cos (\mu), \cos (v)\}$. The eigenvalues of this matrix are principal depolarization factors [17] (depolarization factors along the principal axis given by the eigenvectors of $\mathrm{m}_{\Delta 12345}$ ).

Now, if we consider the matrices generated by the last three generators $\mathrm{J}_{04}, \mathrm{~J}_{05}, \mathrm{~J}_{45}$ of the 
depolarizing part, from the third term of Eq.(20), the eigenvalues of the Mueller matrix $\mathbf{M}_{\Delta 045}$ associated with $\mathrm{T}_{\Delta 045}$ matrix are $\{1,1 / \mathrm{a}, 1 / \mathrm{a}, 1 / \mathrm{a}\}$ after normalization to "a". And we have from Eqs. (17) and the definitions of matrices $\mathrm{L}_{04}(\mathrm{v}), \mathrm{L}_{05}(\mathrm{w})$ and $\mathfrak{R}_{45}(\mathrm{\tau})$ :

$$
\mathrm{a}=\cosh (\mathrm{v}) \cosh (\mathrm{w})
$$

It is worth noting that the result of matrix factorisation leads us to describe the process of depolarization as a combination of an isotropic contraction of the Poincare sphere $\left(\mathrm{M}_{\Delta 045}\right.$ matrix) and an non isotropic contraction ( $\mathrm{M}_{\Delta 12345}$ matrix ) inducing a symmetry breaking.

The corresponding sub-matrices associated with the polarization space verify the following matrix multiplication rules:

$$
\begin{aligned}
\mathrm{T}_{\Delta 12345} \mathrm{~T}_{\Delta 045}=\left(\begin{array}{cc}
\mathrm{M}_{\Delta 12345} & \mathrm{G} \\
\mathrm{H} & \mathrm{J}
\end{array}\right)\left(\begin{array}{cc}
\mathrm{M}_{\Delta 045} & \mathrm{~K} \\
\mathrm{~N} & \mathrm{Q}
\end{array}\right) & =\left(\begin{array}{cc}
\mathrm{M}_{\Delta 12345} \mathrm{M}_{\Delta 045}+\mathrm{GN} & \mathrm{X} \\
\mathrm{Y} & \mathrm{Z}
\end{array}\right) \\
\mathrm{T}_{\Delta 045} \mathrm{~T}_{\Delta 12345} & =\left(\begin{array}{cc}
\mathrm{M}_{\Delta 045} & \mathrm{~K} \\
\mathrm{~N} & \mathrm{Q}
\end{array}\right)\left(\begin{array}{cc}
\mathrm{M}_{\Delta 12345} & \mathrm{G} \\
\mathrm{H} & \mathrm{J}
\end{array}\right)=\left(\begin{array}{cc}
\mathrm{M}_{\Delta 045} \mathrm{M}_{\Delta 12345}+\mathrm{KH} & \mathrm{X}^{\prime} \\
\mathrm{Y}^{\prime} & \mathrm{Z}^{\prime}
\end{array}\right)
\end{aligned}
$$

$\mathrm{M}_{\Delta 12345}$ and $\mathrm{M}_{\Delta 045}$ are matrices of depolarizers, but are not sufficient to define the resulting sub-matrix of the depolarizing part, since the total decomposition takes one of the two following expressions (this is an illustration of the previous remark about Mueller matrix resulting from a product that is not necessary the product of original Mueller matrices):

$$
\begin{aligned}
& \mathrm{M}_{\Delta}^{\mathrm{f}}=\mathrm{M}_{\Delta 12345} \mathrm{M}_{\Delta 045}+\mathrm{GN} \\
& \mathrm{M}_{\Delta}^{\mathrm{r}}=\mathrm{M}_{\Delta 045} \mathrm{M}_{\Delta 12345}+\mathrm{KH}
\end{aligned}
$$

and we have $\mathrm{GN}=\left(\begin{array}{cc}0 & {[0]} \\ {\left[\mathrm{P}_{\Delta}\right]^{\mathrm{T}}} & {[0]_{3}}\end{array}\right)$ and $\mathrm{KH}=\left(\begin{array}{cc}0 & \mathrm{D}_{\Delta} \\ {[0]^{\mathrm{T}}} & {[0]_{3}}\end{array}\right)$ where $[0]_{3}$ is the null matrix of order three. Eventually, the definitions of matrices involved in Eq. (20) and Eq.(26) prove that the sub-matrix $\mathrm{M}_{\Delta}$ has one of the two forms:

$$
\mathbf{M}_{\Delta}^{\mathrm{f}}=\left(\begin{array}{cc}
\mathrm{a} & {[0]} \\
{\left[\mathrm{P}_{\Delta}\right]^{\mathrm{T}}} & \mathrm{m}_{\Delta 12345}
\end{array}\right) \text { or } \quad \mathrm{M}_{\Delta}^{\mathrm{r}}=\left(\begin{array}{cc}
\mathrm{a} & \mathrm{D}_{\Delta} \\
{[0]^{\mathrm{T}}} & \mathrm{m}_{\Delta 12345}
\end{array}\right)
$$

where $\mathrm{m}_{\Delta 12345}$ denotes the symmetric $3 \times 3$ matrix previously introduced and [0] is for $\left[\begin{array}{lll}0 & 0 & 0\end{array}\right]$ vector. After normalization to "a", the three principal depolarization factors of these matrices are :

$$
\left\{\frac{1}{\cosh (\mathrm{v}) \cosh (\mathrm{w})}, \frac{\cos (\mu)}{\cosh (\mathrm{v}) \cosh (\mathrm{w})}, \frac{\cos (v)}{\cosh (\mathrm{v}) \cosh (\mathrm{w})}\right\}
$$

So, the existence of a polarizance vector $\mathrm{P}_{\Delta}$ or a diattenuation vector $\mathrm{D}_{\Delta}$ for the non singular depolarizing matrices is resulting from the interaction between the isotropic contribution and non-isotropic one and depends on the position of the isotropic contraction (or depolarization in optical terms) matrix relative to the non-isotropic one. And the other hand, these both expressions $\mathrm{M}_{\Delta}^{\mathrm{f}}$ and $\mathrm{M}_{\Delta}^{\mathrm{r}}$ correspond with the reverse and 
forward product decomposition concept proposed by Ossikovski \& al. [26] as an answer to the difficulty point out by Morio \& al. [36] about the Lu \& Chipman matrix decomposition process. The "forward" and "reverse" families clearly appear from this two $\mathrm{M}_{\Delta}$ expressions since we have:

$$
M_{D} M_{R} M_{\Delta}^{r}=\left(\begin{array}{cc}
a & D_{\Delta}+D m_{R} m_{\Delta} \\
a D^{T} & D^{T} D_{\Delta}+m_{D} m_{R} m_{\Delta}
\end{array}\right)
$$

where we can always identify $\mathrm{P}^{\mathrm{T}}=\mathrm{D}^{\mathrm{T}}$ as the element of the first column and

$$
M_{\Delta}^{\mathrm{f}} \mathrm{M}_{\mathrm{R}} \mathrm{M}_{\mathrm{D}}=\left(\begin{array}{cc}
\mathrm{a} & \mathrm{aD} \\
\mathrm{P}_{\Delta}^{\mathrm{T}}+\mathrm{m}_{\Delta} \mathrm{m}_{\mathrm{R}} \mathrm{D}^{\mathrm{T}} & \mathrm{P}_{\Delta}^{\mathrm{T}} \mathrm{D}+\mathrm{m}_{\Delta} \mathrm{m}_{\mathrm{R}} \mathrm{m}_{\mathrm{D}}
\end{array}\right)
$$

where we can always identify D as the element of the first row. It is straightforward to demonstrate that a similar result is verified what ever order for $\mathrm{M}_{\mathrm{R}}$ in the product. So, we show that the "reverse" and "forward" decomposition concepts recently introduced [26] may be inferred from the formalism we proposed.

\section{6 - Conclusion}

We proposed a general parametric form for any non singular Mueller matrices. We addressed this problem in a 6 dimension space in order to introduce a group with the same number of degrees of freedom and explained why $S O(5,1)_{\mathrm{e}}$ is a physical admissible solution to solve this question. Generators of this group are used to define possible expressions of an arbitrary non-singular Mueller matrix. The link with previous expressions is established . Eventually, the problem of decomposition of these matrices is addressed and we show that the "reverse" and "forward" decomposition concepts recently introduced [26] may be inferred from the formalism we proposed.

\section{Appendix A:}

We consider the $\mathrm{J}$ matrix defined by :

$$
J=i\left(\sum_{i=1}^{3} \alpha_{i} J_{i 4}+\sum_{k=1}^{3} \beta_{k} J_{k 5}\right)
$$

from the expressions of the generators, $\mathrm{J}$ has the following form:

$$
J=\left(\begin{array}{cc}
{[0]_{4}} & -A^{T} \\
A & {[0]_{2}}
\end{array}\right) \text { with } A=\left(\begin{array}{cccc}
0 & a_{12} & a_{13} & a_{14} \\
0 & a_{22} & a_{23} & a_{24}
\end{array}\right) \quad a_{k l} \in \mathbb{R}
$$

where $[0]_{4}$ and $[0]_{2}$ are respectively for null matrices of 4-dimensions and 2-dimensions. 
A direct computation of $\mathrm{J}^{2}$ gives the following expression:

$$
\mathbf{J}^{2}=\left(\begin{array}{cc}
-\mathrm{A}^{\mathrm{T}} \mathrm{A} & {[0]_{24}^{\mathrm{T}}} \\
{[0]_{24}} & \mathrm{AA}^{\mathrm{T}}
\end{array}\right)
$$

where $\mathrm{A}^{\mathrm{T}} \mathrm{A}$ is a symmetric square matrix with the entries of the first raw and first column equal to 0 . It is straightforward from a recursive computation to prove that the matrices $\mathrm{J}^{2 \mathrm{k}}$ and $\mathrm{J}^{2 \mathrm{k}+1}$ have the following expressions:

$$
\begin{aligned}
\mathrm{J}^{2 \mathrm{k}} & =\left(\begin{array}{cc}
(-1)^{\mathrm{k}}\left(\mathrm{A}^{\mathrm{T}} \mathrm{A}\right)^{\mathrm{k}} & {[0]_{24}^{\mathrm{T}}} \\
{[0]_{24}} & (-1)^{\mathrm{k}}\left(\mathrm{A} \mathrm{A}^{\mathrm{T}}\right)^{\mathrm{k}}
\end{array}\right) \\
\mathrm{J}^{2 \mathrm{k}+1} & =\left(\begin{array}{cc}
{[0]_{4}} & (-1)^{\mathrm{k}+1}\left(\mathrm{~A}^{\mathrm{T}} \mathrm{A}\right)^{\mathrm{k}} \mathrm{A}^{\mathrm{T}} \\
(-1)^{\mathrm{k}}\left(\mathrm{A} \mathrm{A}^{\mathrm{T}}\right)^{\mathrm{k}} \mathrm{A} & {[0]_{2}}
\end{array}\right)
\end{aligned}
$$

The exponential of $\mathrm{J}$ is defined as the usual power series:

$$
\mathrm{e}^{\mathrm{J}}=\sum_{\mathrm{m}=0}^{+\infty} \frac{\mathrm{J}^{\mathrm{m}}}{\mathrm{m} !}
$$

Then, as a summation of symmetric matrices with the entries of the first raw and first column equal to 0 (except for $m=0$ since $J^{0}=I$ ), the resulting sub-matrix of $\mathrm{e}^{\mathrm{J}}$ associated with the polarization space has the form:

$$
\left(\begin{array}{cc}
1 & {[0]} \\
{[0]^{\mathrm{T}}} & \mathrm{m}
\end{array}\right)
$$

where $\mathrm{m}$ denotes a symmetric $3 \times 3$ matrix.

\section{References}

1. R. Sridhar and R. Simon, "Normal form for Mueller matrices in polarization optics", J. Mod. Opt. 41, 1903-1915 (1994)

2. Z. F. Xing, "On the deterministic and non-deterministic Mueller matrix", J. Mod. Opt. 39, 461-484 (1992)

3. V. M. van der Mee, "An eigenvalue criterion for matrices transforming Stokes parameters", J. Math. Phys. 34, 5072-5088 (1993)

4. J. W. Hovenier, "Structure of a general pure Mueller matrix", App. Opt. 33, 8318-8324 (1994)

5. A. V. Gopala, K. S. Mallesh and J. Sudha, "On the algebraic characterization of a Mueller matrix in polarization optics. I. Identifying a Mueller matrix from its $\mathrm{N}$ matrix", J. Mod. Opt. 45, 955-987 (1998)

6. M. S. Kumar and R. Simon, "Characterization of Mueller matrices in polarization optics", Opt. Commun. 88, 464-470 (1992)

7. A. B. Kostinski, C. R. Given and J. M. Kwiatkowski, "Constraints on Mueller matrices of polarization optics", Appl. Opt. 32, 1646-1651 (1993)

8. C.R. Givens and B. Kostinski, "A simple necessary and sufficient condition on physically 
realizable Mueller matrices", J. Mod. Opt. 40, 471-481 (1993)

9. R. Espinosa-Luna, D. Rodrıguez-Carrera, E. Bernabeu, S. Hinojosa-Ruız, "Transformation matrices for the Mueller-Jones formalism", Opt. Int. J. Light Electron.

Opt.,2007,doi:10.1016/j.ijleo.2007.03.008

10. R. Simon, "The connection between Mueller and Jones matrices of polarization optics", Opt. Com. 42, 293-297 (1982)

11. D. G. M. Anderson, R. Barakat, "Necessary and sufficient conditions for a Mueller matrix to be derivable from a Jones matrix", J. Opt. Soc. Am. A 11, 2305-2319 (1994)

12. C. Brosseau, C. R. Givens, A. B. Kotinski, "Generalized trace condition on the MuellerJones polarization matrix", J. Opt. Soc. Am. A 10, 2248-2251 (1993)

13. S.R. Cloude, "Group theory and polarisation algebra", Optik 75, 26-36 (1986)

14. K. Kim, L. Mandel, and E. Wolf, "Relationship between Jones and Mueller matrices for random media", J. Opt. Soc. Am. A 4, 433-437 (1987)

15. J. J. Gil, "Characteristic properties of Mueller matrices", J. Opt. Soc. Am. A 17, 328-334 (2000)

16. J. J. Gil and E. Bernabeu, "Obtainment of the polarizing and retardation parameters of a non-depolarizing optical system from the decomposition of its Mueller matrix", Optik 76, 67-71 (1987)

17. S. Y. Lu, R. A. Chipman, "Interpretation of Mueller matrices based on polar decomposition", J. Opt. Soc. Am. A 13, 1106-1113 (1996)

18. R.Ossikovski, "Interpretation of nondepolarizing Mueller matrices based on singular-value decomposition", J. Opt. Soc. Am. A 25, 473-482 (2008)

19. B. Laude-Boulesteix, A. De Martino, B. Drévillon, L. Schwartz, "Mueller Polarimetric Imaging System with Liquid Crystals", Appl. Opt. 43, 2824-2832 (2004)

20. J. E. Wolfe, R. A. Chipman, "Polarimetric characterization of liquid-crystal-on-silicon panels", Appl. Opt. 45, 1688-1703 (2006)

21. P. Pellat Finet, "Geometrical approach to polarization optics: quaternionic representation of the polarized light", Optik 87, 68-76 (1991)

22. S. Baskal and Y. S. Kim, "de Sitter group as a symmetry for optical decoherence", J. Phys. A 39, 7775-7788 (2006)

23. H. Takenaka, "A unified formalism for polarization optics by using group theory", Nouv. Rev. Opt. 4, 37-41 (1973)

24. Sudha and A.V. Gopala Rao, "Polarization elements, a group theoretical study", J. Opt. Soc. Am. A 18, 3130-3134 (2001)

25. D. Han, Y.S. Kim, M.E. Noz, "Stokes Parameters as Minkowskian Four-vectors", Phys. Rev. E 56, 6065-6076 (1997)

26. R. Ossikovski, A. De Martino, S. Guyot, "Forward and reverse product decompositions of depolarizing Mueller matrices", Opt. Letters 32, 689-691 (2007)

27. S. Sternberg, Group theory and physics, (Cambridge University Press, Cambridge, 1994)

28. J. Bognar, Indefinite Inner Product Spaces, (Springer Verlag, Inc., New York, 1974)

29. I. Gohberg, P. Lancaste and L. Rodman, Matrices and Indefinite Scalar Products, (Biikhauser OT 8, Basel, Boston, 1983)

30. D.H. Sattiger \& O.L. Weaver, Lie Group and Algebras with Applications to Physics, Geometry and Mechanics, (Springer Verlag, Inc., New York, 1991)

31. H.C. van de Hulst, Light Scattering by Small Particles, (Dover Publications, Inc., New York, 1957)

32. K. D. Abhyankar, A.L. Fymat, "Relations between the Elements of the Phase Matrix for Scattering", J. Math. Phy. 10, 1935-1938 (1969)

33. R. Barakat, "Bilinear constraints between elements of the $4 \times 4$ Mueller -Jones transfer matrix of polarization theory", Opt. Com. 38, 159-161 (1981)

34. W. A. Shurcliff, Polarized light, (Harvard University Press, Harvard, 1962)

35. A. A. Sagle, R. E. Walde, Introduction to Lie groups and Lie algebras, (Academic Press, New York, 1973)

36. J. Morio, F. Goudail, "Influence of the order of diattenuator, retarder, and polarizer in polar decomposition of Mueller matrices", Opt. Lett. 29, 2234-2236 (2004) 